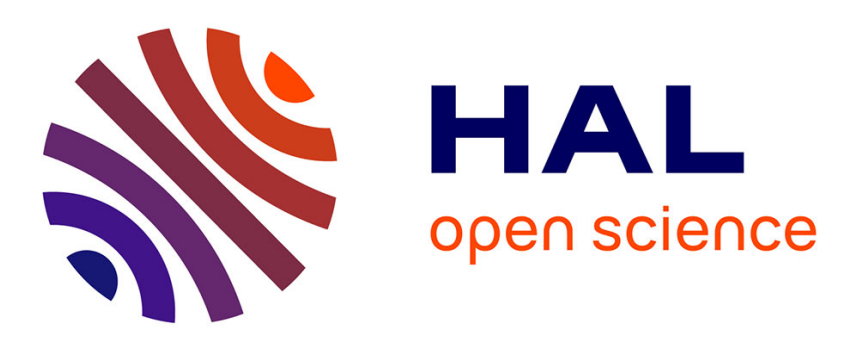

\title{
Entre paysage politique et espace électoral : le canton comme ressource préfectorale de changement du mode de scrutin législatif $(1907$ - 1927)
}

Thomas Marty

\section{- To cite this version:}

Thomas Marty. Entre paysage politique et espace électoral : le canton comme ressource préfectorale de changement du mode de scrutin législatif (1907 - 1927). Lagadec (Yann), Le Bihan (Jean) et Tanguy (Jean François). Le canton. Un territoire du quotidien dans la France contemporaine, Presses Universitaires de Rennes; Presses universitaires de Rennes, pp.211 - 223, 2009, 10.4000/books.pur.100017. hal-00441233

\section{HAL Id: hal-00441233 \\ https://hal.science/hal-00441233}

Submitted on 22 Oct 2020

HAL is a multi-disciplinary open access archive for the deposit and dissemination of scientific research documents, whether they are published or not. The documents may come from teaching and research institutions in France or abroad, or from public or private research centers.
L'archive ouverte pluridisciplinaire HAL, est destinée au dépôt et à la diffusion de documents scientifiques de niveau recherche, publiés ou non, émanant des établissements d'enseignement et de recherche français ou étrangers, des laboratoires publics ou privés. 


\title{
Entre paysage politique et espace électoral : le canton comme ressource préfectorale de changement du mode de scrutin législatif (1907 - 1927)
}

\author{
Thomas Marty \\ ATER de Science politique (IEP Strasbourg) \\ GAP - Paris X Nanterre et GSPE (UMR 7012) - IEP Strasbourg
}

André Siegfried avait posé comme préalable à la rédaction de son Tableau politique en 1913 la double signification électorale du canton. Celui-ci est d'abord l'unité de base du territoire électoral qui permet d'assimiler ce dernier à un véritable espace démographique obéissant à ses propres lois :

C'est le canton qui m'a semblé être l'unité politique la plus naturelle et la plus instructive à observer : il est assez grand pour ne pas entraîner à un détail excessif, et il est en même temps assez restreint pour se prêter à un dessein géographique très souple des opinions politiques ${ }^{1}$.

Secondairement, Siegfried ne voyait pas dans le canton un principe de différenciation des attitudes sous prétexte de votes contradictoires entre l'élection du conseiller général et celle du député :

Dans l'espèce, l'élection cantonale devra être considérée comme d'une moindre valeur révélatrice ${ }^{2}$.

La liaison, même critique, entre toutes les manifestations électorales cantonales paraît donc si évidente que la simple remise en cause de ces liens peut constituer un utile point de départ. Le paysage politique cantonal est d'autant plus susceptible d'être critiqué pour ses inégalités de représentation que l'espace circonscriptionnel qu'il dessine est considéré comme parfaitement lisible et cohérent ${ }^{3}$. Or, le canton n'a cessé d'être perçu par les agents politiques et administratifs eux-mêmes comme une perpétuelle variable d'ajustement ${ }^{4} \mathrm{~d}^{\mathrm{u}}$ un puissant mouvement de réforme électorale législative qui est inauguré vers 1907, au moment ou le scrutin d'arrondissement est vivement critiqué, et s'achève provisoirement en 1927, quand celui-ci est réintroduit comme scrutin de la République.

La focalisation sur cette séquence tient essentiellement à l'évocation du travail préfectoral de re-dimensionnement des circonscriptions législatives. L'introduction possible du scrutin de liste (« départemental») et de la représentation proportionnelle en lieu et place du scrutin uninominal majoritaire d'arrondissement oblige en effet les préfets à un double travail d'expertise : soit de rééquilibrage de la composition cantonale des circonscriptions existantes ; soit de changement total du type de circonscription dans son rapport à l'unité de base cantonale. Autant dire que le canton est plus perçu ici comme un «objet d'étude » susceptible de controverses entre acteurs sociaux que comme un «terrain d'étude » qui favoriserait $a$ posteriori la mesure de telle ou telle inégalité dans la représentation politique ${ }^{5}$. Ce détour par

\footnotetext{
${ }^{1}$ SIEGFRIED André, Tableau politique de la France de l'Ouest, Paris, Imprimerie nationale, 1995 [1 $1^{\text {ère }}$ éd. 1913], p 52.

${ }^{2}$ Ibid., p 51.

${ }^{3}$ Ces inégalités en France sont jugées « sans équivalent dans une démocratie occidentale » (MARTIN Pierre, Les systèmes électoraux et les modes de scrutin, Paris, Montchrestien, 1997, p 148).

4 Voir LAgADEC Yann et LE BIHAN Jean, «L'espace de l'État. Pour une histoire des circonscriptions administratives (Ille-et-Vilaine, 19e siècle) », Annales de Bretagne et des Pays de l'Ouest, 110-4, 2003, p. 81-93.

${ }^{5}$ Sur la représentation comme activité et comme mesure, voir LACROIX Bernard, « La " crise " de la démocratie représentative en France. Éléments pour une discussion sociologique du problème », Scalpel, 1, 1994, p. 6-29.
} 
l'histoire des représentations géographiques communes à des modes de scrutin opposés permet de revenir sur la séparation stricte des formes de territorialité qu'engendreraient inévitablement le scrutin majoritaire et le scrutin proportionnel ${ }^{6}$.

Le matériau convoqué ici résulte d'un ensemble assez méconnu d'une vingtaine de circulaires ministérielles qui, de 1907 à 1927, enjoignent aux préfets d'expertiser tel ou tel mode de scrutin ${ }^{7}$. L'inflation de rapports, de cartes et de tableaux statistiques justifiant le passage de l'une à l'autre de ces modalités invite alors à ne pas tenir pour équivalent ce travail à celui qui voit les gouvernements découper à la marge, et sans véritable consultation préfectorale, les circonscriptions avant chaque élection. L'étude de ces enquêtes préfectorales est d'autant plus cohérente que la stabilité du territoire administratif cantonal sur cette période semble évidente ${ }^{8}$. Il importe au préalable de repérer quelles sont les conditions initiales, à l'époque, qui fondent le sens commun préfectoral à propos de l'influence du canton sur les élections législatives. On s'interrogera alors sur les fonctions historiques et géographiques du canton dans la légitimation de l'ordre politico-territorial. On évoquera ensuite les instruments préfectoraux forgés afin de modifier le rôle électoral du canton : péréquation, sectionnement etc., en prenant soin de spécifier les résultats produits mais aussi les effets pervers parfois tout aussi durables. In fine on pourra s'interroger sur la signification à accorder à cette phase d'intense activité préfectorale. Comment et pourquoi les préfets ont-ils contribué à transformer ainsi certaines conditions du jeu électoral ?

\section{L' « espace » des possibles électoraux : usages historiques et géographiques du canton}

Les interprétations électorales inhérentes à la prise en compte du canton concourent au processus de re-dimensionnement des circonscriptions législatives. La focalisation sur le canton n'a pourtant a priori rien d'évident puisque c'est bien l'arrondissement qui incarne les inégalités de représentation au début des années 1900. Malgré cela, le canton n'apparaît pas spontanément comme le vecteur d'une plus grande justice électorale. Un militant de la cause « proportionnaliste» admet ainsi avant les élections cantonales de 1907 :

Tant que l'on admettra que les assemblées départementales doivent être composées de conseillers généraux à raison d'un par canton, nous ne voyons pas bien le moyen pratique d'arriver à une proportionnalité quelconque. Il faudrait [un] scrutin de liste par arrondissement en spécifiant (...) que chacun des élus représenterait au conseil général un canton déterminé 9 .

Le changement d'échelle qui tend à juger le canton à l'aune des circonscriptions législatives dont il constitue l'unité indivisible autorise ainsi à ne pas considérer la suppression de l'arrondissement comme le seul moyen de remédier aux inégalités électorales. D'autre part, quand la représentation proportionnelle devient un enjeu saillant au cours de la législature 1902-1906, l'injustice électorale qu'elle dénonce est moins celle des territoires que celle des résultats électoraux passés.

\footnotetext{
${ }^{6}$ BUSSI Michel et BADARIOTTI Dominique, Pour une nouvelle géographie du politique : territoire, démocratie, élections, Paris, Anthropos, 2004, p. 111-123.

${ }^{7}$ A travers les archives de neuf départements, notre corpus est constitué ici des rapports préfectoraux à ces circulaires. L'absence de cette source aux Archives Nationales interdit tout raisonnement spatial plus systématique. Cette étude appartient à notre thèse de science politique en cours : Mobilisation politique et analyse électorale au service de l'établissement de la représentation proportionnelle, sous la direction de Bernard Lacroix (université Paris X).

${ }^{8}$ Voir ici même le bilan dressé par Alexandra Laclau, Marie-Vic Ozouf-Marignier et Nicolas Verdier.

9 GAUCHERAND Frédéric, «Un programme d'action pour la représentation proportionnelle », Le Proportionnaliste, 7, 20 Janvier 1907, p. 93.
} 


\section{Cantons à histoire et cantons sans histoire}

C'est donc à partir de la restitution des divisions politiques que peut s'opérer la généalogie des divisions administratives qui concourent à leur donner leur forme la plus explicite. Le canton va vite devenir le point de rencontre entre histoire et géographie comme principes de justification de toute réforme électorale. Il n'est pas convoqué pour son passé politique désormais séculaire mais plutôt pour justifier l'étude à court terme de la succession des différentes élections au conseil général, au conseil d'arrondissement, à la députation. La circulaire du 30 Novembre 1907, qui est pourtant la première à envisager une "péréquation » des circonscriptions électorales, se limite à demander aux préfets une carte de la «nuance politique des conseillers généraux » teintée en trois couleurs (le bleu pour les cantons d'opposition, le rouge pour les cantons républicains, le blanc pour les socialistes unifiés) afin de construire éventuellement de nouvelles circonscriptions législatives à partir de cette base. L'objectif est simple : les élections cantonales passées (été 1904 et 1907) doivent permettre de prédire l'élection législative à venir (1910). Les préfets se montrent plutôt réticents à cet exercice dont l'aspect fictionnel ne leur échappe pas. Le préfet de la Meuse indique par exemple dans son rapport qu'il ne sait pas quelle couleur employer parmi les trois dont il dispose pour deux cantons (Moutiers et Clermont). Dans un brouillon, il propose le bleu (son prédécesseur avait classé ces deux conseillers généraux comme «progressistes ») mais dans ce qui semble être la carte envoyée, les deux cantons sont coloriés en rouge et les deux conseillers généraux classés comme «républicains de gauche ${ }^{10}$ ». Plus réticent encore est le préfet du Rhône qui indique très nettement :

Les cartes ci-jointes ont été dressées en conformité des instructions ministérielles du 30 Novembre 1907. Mais si elles servaient de base à des pronostics concernant les élections législatives, elles risqueraient de devenir une source d'erreurs. En effet la nuance du conseiller général (...) est parfois en contradiction avec le vote législatif du canton ${ }^{11}$.

Le commentaire est d'autant plus surprenant que ce préfet, Charles Lutaud, est assez proche des radicaux et du gouvernement Clemenceau auteur de la circulaire ${ }^{12}$.

\section{L'espace pertinent du canton}

L'imagination cartographique doit alors composer entre cette figuration de l'enchainement chronologique des élections et leur coexistence géographique au sein d'espaces administratifs communs. Les préfets opèrent d'eux-mêmes cette transformation en devançant quelque peu l'incantation ministérielle appelant à mesurer la capacité ultérieure d'un canton à adhérer à la République :

Quant au canton d'Annecy Sud représenté au conseil général depuis les élections dernières par un réactionnaire, il l'est au conseil d'arrondissement par un radical, ce canton ne peut pas être considéré comme absolument mauvais au point de vue politique ${ }^{13}$.

Deux circulaires des 20 et 24 décembre 1907 esquissent les conditions de la création d'une unité de mesure commune au moyen de nouveaux modes de scrutin (aménagement du scrutin uninominal, candidature de liste, représentation dite «proportionnée »... etc.) auxquels on

\footnotetext{
${ }^{10}$ Arch. dép. de la Meuse 3 M 365, 7 décembre 1907.

${ }^{11}$ Arch. dép. du Rhône 3 M 32-33, 9 décembre 1907.

${ }^{12}$ Les données biographiques sur les préfets sont tirées de BARGETON René et al., Dictionnaire biographique des préfets (septembre 1870-mai 1982), Paris, Archives Nationales, 1994.

13 Arch. dép. de Haute-Savoie 3 M 3, novembre 1907. Emile Ténot, le préfet auteur de ce jugement, est un ancien journaliste et publiciste.
} 
pourrait parvenir en élaborant des «groupements de cantons » a priori alternatifs. Les départements qui comportent de grandes agglomérations peuvent voir se confondre un canton très peuplé et un arrondissement. Par exemple, le préfet du Rhône envisage une nouvelle circonscription à partir du $6^{\mathrm{e}}$ arrondissement de Lyon en affirmant :

Le jour où elle serait réduite au $7^{\text {ème }}$ canton de Lyon, la lutte serait plus difficile pour $\mathrm{M}$. Colliard [le député sortant], mais je crois que dans un $2^{\text {ème }}$ tour de scrutin il l'emporterait ${ }^{14}$.

L'accord se fait donc sur le canton comme unité politiquement commensurable même s'il reste en concurrence avec les arrondissements dont la majorité sont maintenus par les radicaux qui entendent ainsi prévenir l'érosion de leur domination préalablement aux élections législatives de 1910. Il est évident que le travail des préfets s'ancre d'abord dans le court terme de la maîtrise des paysages politiques encouragée par les circulaires préélectorales ${ }^{15}$, alors que celles relatives à la réforme électorale sont contraintes par le temps long de l'espace électoral dont il reste à fixer la pertinence circonscriptionnelle. Les effets cumulatifs du travail d'anticipation des préfets en matière d'enquête électorale font qu'ils disposent au début du $\mathrm{XX}^{\mathrm{e}}$ siècle d'une solide expérience ${ }^{16}$. Toutefois, l'imminence d'une réforme électorale ébranle ces certitudes puisque la réélection dépendrait désormais d'un principe différent (voir divergent) de celui qui a présidé à l'élection. On pourrait proposer ici de considérer la «préoccupation cantonale » des préfets comme un avatar du délitement de l'arrondissement au profit du département qui incarnerait de plus en plus le cadre idéal (journalistique, partisan ...etc.) de la mobilisation électorale ${ }^{17}$. De surcroît, la géographie descriptive (statistique, cartographique...) des phénomènes électoraux est à l'époque l'apanage du corps préfectoral qui dispose seul des moyens intellectuels adéquats (possibilité de faire des «enquêtes », possession de cartes et capacité à en dessiner) et qui bénéficie des opportunités politiques de publicisation de ces données ${ }^{18}$.

\section{Les transformations du paysage cantonal : du remaniement au sectionnement des circonscriptions législatives}

Le canton permet de sérialiser les résultats électoraux produits à différents moments dans des circonscriptions administratives distinctes, mais aussi de rendre complètement indivisibles et fongibles les aires qui sous-tendent cette double continuité. Autant de critères que les circonscriptions classiques, l'arrondissement ou le département, ne semblent pas réunir à elles seules, au moins aux yeux des préfets. L'arrondissement est considéré comme le facteur territorial et anamorphosé des inégalités de représentation, tandis que l'on envisage parfois le dépassement du département dans des circonscriptions «régionales ${ }^{19}$. La typologie des instruments préfectoraux qui ont permis en deux décennies de réévaluer la position du canton

\footnotetext{
${ }^{14}$ Arch. dép. du Rhône 3 M 32-33, janvier 1908.

${ }^{15}$ PHELIPPEAU Éric, «Conjonctures électorales et conjectures préfectorales. Le vote et la formation d'un savoir politico-administratif », Scalpel, 1, 1994, p. 52 - 73.

16 VoILliot Christophe, La candidature officielle. Une pratique d'Etat de la Restauration à la Troisième République, Rennes, Presses universitaires de Rennes, 2005, p. 169-181.

${ }^{17}$ La consécration historiographique et les limites de cette départementalisation de la vie politique ont été soulignées par ROUGERIE Jacques, «Faut-il départementaliser l'histoire de France », Annales. Economies, Sociétés, Civilisations, 21, 1, 1966, p. 178 - 193.

${ }^{18}$ Sur l'offre de cartographie électorale au $\mathrm{XIX}^{\mathrm{e}}$ siècle et la place prise par les préfets à partir des années 1880 , voir GARRIGOU Alain, «Invention et usages de la carte électorale », Politix, 10-11, 1990, p. 33-44.

19 Jean Jaurès propose un amendement destiné à introduire «l'utilisation inter-départementale des " restes électoraux " » (Journal Officiel, Débats parlementaires, 24 Janvier 1912, p 65. et s.).
} 
indique deux voies à suivre. Tout d'abord, la remise en cause de la topographie des arrondissements comme base de la représentation législative (en 1907 et 1908 puis essentiellement entre 1924 et 1927) débouche sur les différentes modalités d'un simple remaniement des circonscriptions autrement appelé «péréquation ». Ensuite, l'hypothèse du scrutin de liste et les variantes de l'équation proportionnelle entre voix et sièges annoncent les projets de sectionnement du département lui même (entre 1911 et 1913 ainsi qu'en 1919).

\section{Le canton et l'arrondissement : l'invention de la péréquation}

Le remaniement des circonscriptions électorales dans le cadre du mode de scrutin existant n'apparaît pas spontanément avec ces circulaires de réforme électorale. Depuis la loi électorale de 1889, qui rétablit ce scrutin uninominal d'arrondissement après la parenthèse «boulangiste» du scrutin de liste, chaque élection ou presque était précédée d'une loi modifiant à la marge le tableau des circonscriptions. La faible part préfectorale dans ce travail de «découpage » s'explique en partie par la prise en compte persistante de l'arrondissement comme base de ce scrutin majoritaire. A partir de 1907, l'implication de l'expertise préfectorale est toute autre puisqu'elle doit répondre à une volonté de réforme aux objectifs et dénominations variables. Au-delà de cette diversité, c'est bien le principe de la péréquation qui émerge, tentant de faire de chaque député le représentant d'un nombre d'habitants à peu prés égal. Le canton apparaît invariablement comme l'unité territoriale capable de modifier la composition des circonscriptions législatives. Les données morphologiques des cantons qui sont appelés à changer de circonscription appellent toutefois une réserve. Dans la Creuse, de 1908 à 1927, les quatre projets de remaniement des arrondissements atteignent toujours le même taux d'électeurs inscrits (environ 30 à $32 \%$ ) et, bien que cela n'ait rien d'évident $a$ priori, une proportion de votants également stable $(72 \text { à } 75 \%)^{20}$. On pourrait ainsi émettre l'hypothèse que les préfets ne peuvent plus agir que sur les seuls équilibres partisans dont la logique classificatoire évolue pourtant plus vite que la géographie et la démographie des opinions considérées comme relativement stables (hormis la parenthèse de la Première Guerre et ses conséquences).

Quelque floues qu'elles soient quant à leurs objectifs politiques, les circulaires permettent l'émergence de principes de base à respecter comme celui de «l'intégrité cantonale ». Dans la circulaire du 20 Décembre 1907, le critère n'est pas explicitement posé, mais cela n'empêche pas les préfets de l'anticiper :

Je me suis efforcé, sans pouvoir y parvenir toutefois complètement, de respecter les limites des cantons. Je ne déroge, d'ailleurs, au principe de l'intégrité cantonale que dans la ville de Lyon, c'est-à-dire là où le fractionnement des cantons ne comporte aucun inconvénient ${ }^{21}$.

Lors du rétablissement du scrutin uninominal dans les années 1920, les circulaires affirmeront à nouveau ce principe en invoquant cette fois l'obligation de respecter le tracé de "cantons entiers et limitrophes ». Si les cantons sont maintenus dans leur intégrité, leur redistribution électorale est encadrée par certains principes géographiques, économiques et politiques que les préfets accueillent avec circonspection, comme l'indique l'argumentaire/contreargumentaire de P. Lardin de Musset dans la Loire en 1908 :

Les circonscriptions sont délimitées non seulement par affinités de cantons mais aussi en tenant compte des considérations économiques, agricoles et industrielles ${ }^{22}$.

\footnotetext{
${ }^{20}$ Arch. dép. de la Creuse 3 M 268.

${ }^{21}$ Arch. dép. du Rhône 3 M 32-33, décembre 1907.

${ }^{22}$ Arch. dép. de la Loire 3 M 206, 18 janvier 1908. Ce préfet est le neveu d'Alfred de Musset.
} 
Le préfet de la Sarthe exprime quant à lui, toujours en 1907, des réticences plus qu'importantes pour justifier le retrait du canton de Sillé-le-guillaume, jugé trop peu urbain, de la $2^{\mathrm{e}}$ circonscription du Mans :

Il n'est pas douteux que les communications de ce canton avec son nouveau chef lieu d'arrondissement seront beaucoup plus longues et difficiles. Mais il ne m'a pas paru possible de constituer autrement les nouvelles circonscriptions... ${ }^{23}$.

L'étau dans lequel est pris le canton se desserre tout à fait lorsque l'arrondissement lui-même est appelé à éclater. La force du canton est justement de pouvoir y survivre, même si cette capacité n'est pas équitablement partagée. Le préfet de Haute Savoie indique par exemple :

Le remaniement des circonscriptions actuelles en vue de la suppression de l'une d'elles m'a paru comporter nécessairement la disparition de l'arrondissement de Saint Julien, le seul dont les cantons puissent être logiquement attribués aux arrondissements voisins ${ }^{24}$.

\section{Le canton et le département : contrainte et atout du sectionnement}

Le maintien de l'horizon pratique de la candidature uninominale et du principe majoritaire lie inévitablement le sort du canton à celui de l'arrondissement. Il en va tout autrement lorsque le scrutin majoritaire est condamné par l'introduction possible de la représentation proportionnelle. Le canton doit alors faire face au département tout entier (scrutin de liste) et non plus à ses subdivisions. Pour les départements les plus peuplés, on ne parle plus de «péréquation des circonscriptions » mais de «sectionnement du département ». La virginité de cet instrument semble totale : on envisage de repartir des divisions cantonales elles-mêmes en ne faisant aucune mention de l'arrondissement. Le sectionnement sera surtout pratiqué par les circulaires de 1911 et 1913, c'est-à-dire au moment où le Parlement débat de la proportionnelle ${ }^{25}$. Les critères de mesure déployés sont différents : le canton n'est plus seulement une ressource aux usages variables mais devient alternativement et contradictoirement une contrainte (notamment sur le plan économique et démographique) et un atout stratégique (sur le plan purement politique).

La réduction du nombre de circonscriptions, et donc corrélativement leur élargissement, bouleversent les prévisions électorales fondées jusqu'ici sur l'arrondissement et son offre politique peu sujette au renouvellement. Evoquant en 1908 l'arrondissement de Thonon, le préfet de Haute Savoie remarque :

La personnalité très sympathique de Mr Mercier [le député] entraîne un certain nombre de voix de modérés sur lesquelles il ne pourrait plus compter avec le scrutin de liste ${ }^{26}$.

La tâche est d'autant plus délicate que les critères du sectionnement varient d'une circulaire à l'autre. S'il concerne généralement des départements devant élire plus de cinq ou six députés, sa base démographique varie aussi : en 1907 et 1911, un député doit être élu pour 70000 ou

\footnotetext{
${ }^{23}$ Arch. dép. de la Sarthe 3 M 418, 4 janvier 1908. Ce préfet, Jules d'Auriac s'est essayé - à la même époque -à la compréhension d'un espace plus « large » dans Etudes sociologiques. La France d'aujourd'hui et la France de demain (Paris, Berger-Levrault, 1908).

${ }^{24}$ Arch. dép. de la Haute-Savoie 3 M 3, 23 décembre 1907.

${ }^{25}$ MARTY thomas, «La Chambre des Députés comme ressource et comme théâtre : la consécration de la cause proportionnaliste par le travail en Commission du suffrage universel », dans COHEN Antonin, LACROIX Bernard et RIUTORT Philippe, Les formes de l'activité politique. Eléments d'analyse sociologique XVIIIè - Xxè siècle, Paris, PUF, 2006, p $83-101$.

${ }^{26}$ Arch. Dép de la Haute Savoie, 3 M 3, avril 1908. Ce préfet, Léon Pommeray, avait lui même remporté deux élections législatives (en 1897 et 1898) avant d'en perdre une troisième (en 1902).
} 
75000 habitants alors qu'en 1913, le seuil est de un député pour 21500 électeurs inscrits. En d'autres termes, l'étendue de la circonscription visée empêche que le canton puisse avoir les mêmes potentialités réformatrices qu'il possède en régime de péréquation. Cela est particulièrement vrai dans les départements les plus peuplés, comme en Gironde : si les projets de péréquation permettent de déplacer plus d'une dizaine de cantons (et d'en diviser quelques-uns), soit entre 35 et $40 \%$ de la population départementale ${ }^{27}$, les pistes de sectionnement n'envisagent le déplacement que d'un ou deux cantons $(1,3 \%$ à $4,4 \%$ de la population $)^{28}$. Les effets des projets de sectionnement sur la sélection des «bons » ou des «mauvais » cantons sont pourtant réels et sont attestés par la continuité administrative du travail des préfets successifs. Ainsi, en Gironde, le dernier projet de péréquation de 1927 n'agit que sur un canton, celui d'Audenge, qui était précisément le seul point de bascule autour duquel s'articulait le sectionnement du département en $1911^{29}$.

Ces contraintes sont toutefois réversibles et l'étendue des circonscriptions nouvelles - des départements entiers ou des moitiés de département - favorise alors l'anticipation de bénéfices électoraux risqués mais exponentiels. L'inflation des modalités de sectionnement (plus nombreuses que pour la péréquation) accentue la croyance préfectorale dans les vertus de la concurrence électorale. Est-ce à dire que les enquêtes prévoyant le sectionnement auraient une finalité plus stratégique que celles qui visaient la péréquation ? Tel jugement du préfet de la Gironde, qui évoque les raisons du retrait du septième canton de Bordeaux d'une circonscription qui recouvrerait toute l'agglomération, tend à accréditer cette hypothèse :

Ce $7^{\text {ème }}$ canton - isolé des 6 autres - est le boulevard du socialisme unifié ; la députation de Bordeaux devant être de l'opposition, il est préférable qu'elle soit réduite au strict minimum ${ }^{30}$.

Ce type d'enquête présente en tout cas des moyens inédits de mobilisation pré-électorale entre les mains des préfets qui peuvent contraindre par exemple les républicains d'un même département à s'unir et ainsi se jouer un peu plus des spécificités cantonales qui désormais paraissent solubles dans la départementalisation du jeu politique. Cette extension de la lutte électorale ne répond d'ailleurs pas seulement à des facteurs de développement des entreprises politiques qui seraient désormais «départementalisées». Les transformations économiques d'avant-guerre et la nature des problèmes de reconstruction dans les régions dévastées poussent aussi à une telle cristallisation des enjeux. Ces raisons mènent paradoxalement le gouvernement Clemenceau à se passer des préfets lors du seul sectionnement effectif qui accompagne l'adoption partielle d'une forme de représentation proportionnelle par la loi du 12 Juillet 1919. C'est au niveau gouvernemental et avec l'aide de la commission (parlementaire) du suffrage universel que les hypothèses de sectionnement sont étudiées ${ }^{31}$. Les députés sortants remplacent temporairement les préfets comme agents garantissant l'unité du département !

\footnotetext{
${ }^{27}$ En 1907 : 16 cantons et 3 communes changent de circonscription (39, $4 \%$ de la population). En $1925: 15$ cantons et 5 communes $(40,4 \%)$ pour le premier projet et 13 cantons et 4 communes pour le second $(36,3 \%)$ (arch. dép. de la Gironde, 3 M 112 et 3 M 116).

${ }^{28}$ Ibid. 3 M 113, septembre 1911.

${ }^{29}$ Ibid. 3 M 116, 23 janvier 1927, 3 M 113, septembre 1911.

${ }^{30}$ Ibid. 3 M 113, avril 1913.

${ }^{31}$ Arch. Nat. C 7721, 29 juillet au 4 septembre 1919.
} 


\section{Les préfets au travail : entre expertise électorale et administration des affaires politiques}

La mise en cause du rôle politique du préfet, trop souvent perçu comme un agent d'influence, permet d'aborder quelques-unes des raisons de la constitution d'un répertoire d'action administratif inédit et relativement en avance sur la professionnalisation du corps préfectoral qui n'apparaît que plus tard dans le $\mathrm{XX}^{\mathrm{e}}$ siècle $^{32}$. L'action préfectorale n'aboutit pas qu'à l'uniformisation d'une unité de mesure, elle génère aussi une multiplication des interdépendances politiques, que le canton ne fait que rendre visibles avant d'être dépassé par elles. Il importe donc de s'attarder sur les interactions entretenues par les préfets engagés dans la production de cette expertise électorale non seulement avec le personnel politique local mais également avec les instances parlementaires et gouvernementales chargées d'impulser puis de répercuter ces rapports préfectoraux ${ }^{33}$.

\section{Simulations et dissimulation}

Les préfets réalisent l'interface auprès des électeurs et de leurs candidats auxquels ils présentent les simulations théoriques inventées ou importées par des publicistes ou des parlementaires. Il n'y a pas jusqu'au recours systématique aux sous-préfets qui ne soit atteint par cette division du travail tâtonnante. Ceux dont l'arrondissement comporte plusieurs circonscriptions législatives sont particulièrement enclins à concurrencer le préfet et à affirmer l'immuabilité de tel ou tel canton. Le plus souvent, toutefois, c'est en direction du gouvernement qu'il est délicat de communiquer les contingences politiques locales. En 1925, le préfet de la Marne prévient par exemple le ministère Herriot que le retour du scrutin uninominal ne peut se faire sans un certain réalisme :

Quel que soit le découpage auquel il sera procédé dans le département, des objections seront toujours présentées (...) il semble difficile de rattacher à l'arrondissement de Sainte Menehould le canton le plus proche de l'arrondissement de Reims sans se heurter aux réclamations de conseillers généraux parlementaires qui peuvent invoquer des considérations géographiques ${ }^{34}$.

La collaboration étroite avec le personnel républicain, le plus souvent radical avant la guerre, est dissimulée dans les rapports eux-mêmes sauf quand elle parvient justement à s'extraire de l'influence personnelle (ou partisane) et à se prévaloir d'un bon ancrage territorial :

Ces chiffres ont été arrêtés par moi, d'accord avec certaines personnalités républicaines, au courant des situations politiques de chaque canton et dont le jugement est le plus impartial ${ }^{35}$.

Ces indications sont encore plus courantes dans les rapports des sous-préfets en contact régulier avec les élus cantonaux même si après la guerre une plus grande abstraction des enquêtes pré-électorales tend à effacer ces intermédiaires dans le sens promu, par exemple, par le décret-loi Poincaré du 26 Septembre 1926 portant suppression de 106 sous-préfectures.

\footnotetext{
${ }^{32}$ Voir les pistes avancées en ce sens par TANGUY Gildas, « Les préfets face à la grève : faire-savoir, savoir-faire et " expertise de gouvernement ". Autour des pratiques de résolution des conflits sociaux (1880-1914) », in AUdREN Frédéric, LABORIER Pascale, NAPOli Paolo, Vogel Jakob (dir.), Les sciences camérales : activités pratiques et histoire des dispositifs publics, Paris, Presses universitaires de France, coll. CURAPP, 2006 (à paraître).

${ }^{33}$ MARTY Thomas, «Le changement du mode de scrutin entre le temps de l'élection et celui de la réélection : mobilisations politiques et expertises préfectorales », Pôle Sud, 27, 2007 (à paraître).

${ }^{34}$ Arch. dép. de la Marne 2 M 30, $1^{\text {er }}$ avril 1925.

${ }^{35}$ Arch. dép. de la Haute-Marne, M 1207, décembre 1905.
} 


\section{Le canton dans la transformation de la compétition électorale}

La dimension la plus visible du rapport de forces dans lequel sont pris les préfets est celle qui les oppose aux instances nationales en charge de mener à bien les projets de réforme électorale qui animent leurs majorités successives et parfois opposées. Il est bien délicat de savoir ce que deviennent les rapports préfectoraux. Quelques circulaires additionnelles tentent de remédier à la qualité décevante de certains rapports comme celle du 20 février 1925 qui indique :

Les rapports qui m'ont été adressés, établis trop rapidement, présentaient entre eux des divergences de méthode considérables et n'avaient pu être précédés des recherches minutieuses et des consultations que nécessite un problème de cette nature.

En 1927, signe des temps nouveaux, le ministre de l'Intérieur Albert Sarrault invite pour la première fois les préfets à se rendre au ministère de l'Intérieur pour examiner leurs propositions au cours d'une réunion, cartes et tableaux à l'appui. Une seule fois au cours de ces deux décennies un dialogue semble s'être amorcé entre les préfets et un groupe de parlementaires, essentiellement des radicaux, le Groupe Parlementaire de Défense Républicaine contre la Représentation Proportionnelle. Cette collaboration officieuse favorisa les projets du député haut-marnais Arthur Dessoye en mettant à sa disposition les résultats préfectoraux produits à partir des circulaires «clémencistes » de décembre 1907 et janvier 1908. Contre-feux allumés pour retirer aux «proportionnalistes » le monopole de la réforme, ces projets irriguent la controverse tout au long de la législature 1910-1914 pour imposer l'objectif de la "péréquation ». Pénétré de ces principes, le gouvernement rappelait sans cesse celui de l'intégrité cantonale :

La commission a établi en principe que le canton devait être substitué à l'arrondissement comme unité électorale, que ses limites actuelles devaient en conséquence être toujours respectées et que les circonscriptions ne devaient comprendre que des cantons entiers et limitrophes ${ }^{36}$.

Aucun préfet n'avait osé théoriser de manière aussi radicale le nouveau rôle électoral du canton. Si la plupart d'entre eux modifièrent leurs propositions sans broncher, certains entrèrent dans une guerre ouverte dans laquelle le canton tenait lieu de pomme de discorde. Charles Lutaud, préfet du Rhône, indiquait par exemple qu'il avait effectivement dérogé au principe de l'intégrité cantonale, mais seulement afin de «respecter les habitudes de la population lyonnaise qui a coutume d'élire les députés par arrondissement et non par canton $^{37}$ ». Dans le jeu troublé de l'après-guerre, les cartes sont ainsi largement redistribuées par rapport aux ambitions initiales conçues tant par les préfets que par certains élus. Le préfet de la Marne note par exemple que la composition cantonale des circonscriptions ne joue plus selon lui qu'en troisième position derrière la notoriété des candidats et celle des partis ${ }^{38}$. La lutte pour la définition du sens politique accordé au canton tourne alors ostensiblement à la mise en avant de la connaissance électorale locale, celle des «hommes », de leurs réseaux et

\footnotetext{
${ }^{36}$ Arch. dép. du Rhône, 3 M 32-33, 19 février 1908. La diffusion de cette circulaire aux autres préfectures n'est pas vérifiée.

${ }^{38}$ Arch. dép. de la Marne 2 M 30, mars 1922. Sur la rationalisation de la vie politique dans une double projection spatiale (départementale) et temporelle (maîtriser la réélection), voir PHELIPPEAU Éric, L'invention de l'homme politique moderne. Mackau, l'Orne et la République, Paris, Belin, 2002, p. 272 et s. Pour une comparaison contemporaine et internationale, voir MoRgENSTERN Scott et POTHOFF Richard F., "The Components of elections : district heterogeneity, district-time effects, and volatility », Electoral Studies, 24, 2005, p. 17-40.
} 
de leurs motivations, dont les préfets prétendent avoir le monopole. Le nouveau système électoral adopté en 1919 traduit le hiatus entre les différents intérêts réformateurs : la départementalisation du scrutin construite sur le respect des arrondissements déjà existants définit ce compromis qui tente de concilier les ambitions de justice dans la représentation et les réalités de l'espace circonscriptionnel dont le canton est la mesure. Le travail préfectoral tel qu'on a tenté de le restituer n'est sans doute pas circonscrit à la défense de ce paysage politique local, il participe aussi à la transformation des formes de la compétition politique. Les perceptions du canton elles-mêmes se confondent dans ce double processus en dissuadant certains candidats ou en en incitant d'autres à se présenter dans l'espoir qu'un changement de circonscription puisse modifier profondément le cours des choses.

Thomas Marty

thomarty@hotmail.com

3 , rue du Muguet

67640 Fegersheim. 